Scientific Investigation in Dentistry - SID

Artigo original

\title{
ANÁLISE DO CONHECIMENTO DE PROFISSIONAIS DE NÍVEL SUPERIOR DA UNIDADE DE SAÚDE DA FAMÍLIA SOBRE O CÂNCER BUCAL
}

\section{ANALYSIS OF THE KNOWLEDGE OF HIGHER EDUCATION PROFESSIONALS FROM THE FAMILY HEALTH UNIT ON ORAL CANCER}

\begin{abstract}
Amanda Barroso da SILVA ${ }^{1}$, Danielle Batista de SOUZA , Gabriel Carneiro de OLIVEIRA ${ }^{1}$, Gabrielly Caetano de SOUZA ${ }^{1}$, Mayara Barbosa Viandelli MUNDIM², Liliane Braga Monteiro dos REIS ${ }^{2}$

\footnotetext{
${ }^{1}$ Acadêmico(a) do Curso de Odontologia, Universidade Evangélica de Goiás - UniEVANGÉLICA, GO, Brasil.

${ }^{2}$ Doutora em Odontologia pelo Programa de Pós-Graduação em Odontologia da Faculdade de Odontologia da Universidade Federal de Goiás.
} Professora Adjunta do Curso de Odontologia da Universidade Evangélica de Goiás.
\end{abstract}

Informação sobre o manuscrito

Recebido em: 18 Jun 2021

Aceito em: 02 Dez 2021

Fonte de fomento da pesquisa: FUNADESP

\author{
Autor para contato: \\ Liliane Braga Monteiro dos Reis \\ Avenida Universitária Km 3,5 Cidade Universitária, \\ Anápolis, GO, Brasil - 75070290 \\ Telefone: (62) 33106602 - Fax: (62) 33106607 \\ E-mail: lilianeprofessora@yahoo.com.br
}

\begin{abstract}
RESUMO
Objetivo: Analisar o conhecimento dos cirurgiões-dentistas, médicos e enfermeiros das Unidades de Saúde da Família de Anápolis-GO, a respeito do câncer bucal. Métodos: Estudo quantitativo com médicos, enfermeiros e cirurgiõesdentistas das equipes de saúde da família do município de Anápolis-GO. A coleta de dados ocorreu por meio do aplicativo Google Forms. Foram coletados dados sobre o perfil demográfico, conhecimento sobre prevenção, diagnóstico e tratamento e atitudes e práticas frente ao tema. Os dados foram coletados entre 11/2020 e 02/2021, tabulados e analisados por meio de estatística descritiva, utilizando-se o programa IBM SPSS 22,0. Parecer CEP UniEVANGÉLICA número 3.848.550. Resultados: Taxa de resposta de 11,6\% ( $\mathrm{N}=25), 80 \%$ sexo feminino, idade média de 36,4 anos (DP=12,0), tempo de graduação entre zero e 34 anos. $60 \%(n=15)$ consideram seu nível de conhecimento sobre o câncer bucal como ótimo ou bom. Quanto a maior prevalência $80 \%(n=20)$ citou o sexo masculino, com idade acima de 40 anos $76 \%(n=19)$ e $40 \%(n=10)$ respondeu que o carcinoma de células escamosas é a neoplasia mais prevalente na boca. $20 \%(n=5)$ dos participantes responderam não saber sobre as características clínicas mais prevalentes compatíveis com a hipótese de câncer de boca e a maioria $76 \%(n=19)$ não se sente capacitado para realizar uma biópsia. Grande parte dos participantes $88 \%(n=22)$ expressou a vontade de participar de algum curso de atualização sobre o tema. Conclusões: Os profissionais relatam conhecimento sobre o câncer de boca embora se sintam inseguros diante de algumas especificidades, e expressam vontade de capacitação sobre o tema.
\end{abstract}

PALAVRAS-CHAVE:

Câncer Bucal. Conhecimento. Profissional de Saúde. Atenção Primária à Saúde. 


\section{INTRODUÇÃO}

O câncer bucal é uma das neoplasias malignas mais comuns do segmento cabeça e pescoço, e que mais causam mortalidade, ou quando não levam ao óbito, promovem mutilações e trazem sérios danos psicológicos para o paciente e seus familiares. O diagnóstico tardio do câncer de boca é a principal causa de morbidade da doença, ${ }^{1}$ e devido à incidência de casos faz com que este se torne um problema de saúde pública. ${ }^{2}$ Apesar do crescente número de estudos e informações, essa neoplasia ainda passa despercebida pelo olhar de muitos profissionais da área da saúde. Aliando a falta de sintomatologia no estágio inicial, o medo da população e a falta de preparo dos profissionais são fatores que impossibilitam o diagnóstico precoce da doença, trazendo grandes desafios para a sua cura. ${ }^{3}$

O câncer bucal acomete mais homens acima de 45 anos, que na maioria das vezes, estão expostos a fatores de riscos relevantes, como tabagismo, etilismo e exposição excessiva ao sol. ${ }^{4} \mathrm{~A}$ língua e soalho bucal são considerados os sítios mais acometidos, mas também poderá apresentar-se na gengiva, mucosa jugal, mucosa labial e palato duro. Dentre as neoplasias orais o carcinoma epidermoide, também chamado de carcinoma de células escamosa ou espinocelular, caracteriza o câncer de cavidade oral mais comum, representando mais de $90 \%$ dos casos diagnosticados. ${ }^{5}$

Segundo as estatísticas do Instituto Nacional de Câncer (INCA), a estimativa para novos casos de câncer de cavidade oral no Brasil em 2020 são de 15.190, sendo 11.180 homens e 4.010 mulheres. ${ }^{6}$

Para o profissional que atua na atenção básica onde o primordial é a prevenção e promoção da saúde, é fundamental que saiba orientar o paciente sobre os fatores de risco, como também deve saber diagnosticar precocemente esta patologia. $^{7}$

Estudos realizados anteriormente no Brasil, mostraram que os cirurgiões-dentistas se autoavaliam com baixo conhecimento e inseguros para realizar procedimentos de diagnóstico do câncer de boca. 2,8,9 Jnaneswar et al. (2017), ${ }^{10}$ Tomé Júnior et al. (2019) ${ }^{11}$ e Santos et al. (2010) $)^{12}$ apontam em suas pesquisas, que grande parte dos pacientes buscam preferencialmente realizar uma consulta médica quando constatam apresentar uma lesão na boca, e posteriormente, procuram a assistência odontológica. Sendo assim, de suma importância o conhecimento sobre o câncer bucal, tanto por parte do cirurgião-dentista, quanto de todos os profissionais da atenção básica. ${ }^{13}$

Portanto, este estudo analisou o conhecimento dos cirurgiões-dentistas, médicos e enfermeiros das Unidades de 
Saúde da Família, na cidade de Anápolis-GO, a respeito do câncer bucal.

\section{MATERIAL E MÉTODOS}

Foi realizado um estudo observacional, descritivo, transversal, com profissionais médicos, enfermeiros e cirurgiões-dentistas que compõem as equipes de saúde da família do município de Anápolis-GO, previamente aprovado pelo Comitê de Ética em Pesquisa sob o parecer número 3.848.550.

$\mathrm{O}$ instrumento de coleta de dados foi um questionário autoaplicável utilizando-se o aplicativo de gerenciamento de pesquisas do Google, o Google Forms. Foi enviado à Secretaria de Saúde um link juntamente com um texto explicativo sobre a pesquisa para envio aos profissionais médicos, enfermeiros e cirurgiões-dentistas das equipes de saúde da família. O link permitia acesso ao Termo de Consentimento Livre e Esclarecido (TCLE) e em caso de aceite o acesso ao questionário. Em função da baixa taxa de resposta foi também realizado contato telefônico com as Unidades de Saúde, no qual foi também encaminhado para um ou mais profissionais das equipes o mesmo texto e o link de acesso ao Google Forms. O questionário foi composto por 30 questões objetivas, já utilizadas por Vasconcelos $(2006)^{14}$ e adaptadas para esta pesquisa, com perguntas sobre o perfil demográfico e o conhecimento dos profissionais sobre prevenção, diagnóstico, tratamento e atitudes e práticas frente ao câncer bucal.

O período de coleta de dados foi entre novembro de 2020 e fevereiro de 2021. Os dados obtidos foram tabulados e analisados por meio de estatística descritiva, utilizandose o programa IBM SPSS 22.0.

\section{RESULTADOS}

Segundo a Secretaria Municipal de Saúde de Anápolis-GO, o município possui 44 Unidades de Atenção Básica de Saúde, totalizando 72 equipes de Saúde da Família, sendo que todas têm equipe de saúde bucal, totalizando aproximadamente 216 profissionais entre médicos, enfermeiros e cirurgiões-dentistas. Destes, aceitaram participar da pesquisa 25 profissionais, sendo 16 cirurgiões-dentistas, sete médicos e dois enfermeiros (taxa de resposta de 11,6\%).

Quanto a avaliação do perfil demográfico do grupo estudado, a maioria declarou ser do sexo feminino (80\%) e apresentavam faixa etária entre 25 a 60 anos, com idade média de 36,4 anos ( $D P=12,0$ ). Com relação ao tempo de graduação, os participantes tinham de 0 a 34 anos de formação, sendo que $84 \%$ haviam concluído sua formação em instituição privada e $88 \%$ afirmaram obter alguma especialização (Tabela 1).

Analisando a autopercepção dos profissionais, verificou-se que a maioria dos cirurgiões-dentistas consideram o seu nível 
de conhecimento sobre o câncer bucal como ótimo ou bom e os demais profissionais a maior parte considerou o conhecimento entre regular ou ruim (Tabela 2 ).

Quanto a prevalência do câncer bucal, observou-se que $80 \%$ profissionais afirmaram ser maior no gênero masculino. Quanto ao tipo de neoplasia mais prevalente na boca os profissionais se dividiram entre o carcinoma de células escamosas e outras neoplasias. A idade acima de 40 anos foi a mais mencionada (76\%) como faixa etária de maior prevalência do câncer bucal (Tabela 2).

Tabela 1 - Distribuição da amostra segundo variáveis demográficas e sobre o exercício da profissão. Anápolis-GO, 2021. ( $\mathrm{N}=25)$

\begin{tabular}{lrr}
\hline \multicolumn{1}{c}{ Variável } & $\mathbf{n}(\%)$ & Média (DP) \\
\hline Sexo & $20(80)$ & \\
Feminino & $5(20)$ & \\
Masculino & & \\
Faixa etária (anos) (N=24) & $12(50)$ & $36,4(12,0)$ \\
$25-34$ & $12(50)$ & \\
$35-60$ & & $12,9(8,9)$ \\
Tempo de graduação (anos) & $11(44)$ & \\
$0-8$ & $14(56)$ & \\
$13-34$ & $16(64)$ & \\
Ocupação & $2(8)$ \\
Cirurgião-dentista & $7(28)$ \\
Enfermeiro & \\
Médico & $4(16)$ \\
Instituição da Graduação & $21(84)$ \\
Pública & \\
Privada & $22(88)$ \\
Especialização & $3(12)$ \\
Sim & \\
Não &
\end{tabular}

Questionados sobre as características clínicas mais prevalentes que podem ser compatíveis com a hipótese de câncer de boca, $44 \%$ profissionais afirmaram que a lesão se apresenta de forma infiltrativa, bordas endurecidas e crescimento rápido, importante ressaltar que $20 \%$ não souberam responder à pergunta (Tabela 2 ).

Acerca da capacidade de identificar as lesões potencialmente malignas, $40 \%$ dos participantes avaliaram como bom e os demais como regular ou ruim (Tabela 2).

Com relação a prática clínica, $60 \%$ participantes afirmaram orientar apenas os pacientes com algum fator de risco predisponente ao câncer bucal, sendo que $24 \%$ orientam todos os pacientes, independente de apresentarem algum dos fatores para o desenvolvimento da doença. Quanto ao diagnóstico do câncer de boca mais da metade dos participantes (52\%) informaram já ter realizado esse diagnóstico (Tabela 3).

Sobre se sentir capacitado para realiza uma biópsia a maioria (76\%) discorda, incluindo alguns cirurgiõesdentistas. Quanto ao tipo de biópsia a ser realizada em lesão suspeita de câncer de boca em tecido mole apenas os cirurgiões dentistas responderam, e divergiram quanto ao procedimento entre incisional e excisional (Tabela 3). 
Tabela 2 - Conhecimentos a respeito do câncer bucal. Cirurgião-dentista, Enfermeiro e Médico. Anápolis-GO, 2021. $(\mathrm{N}=25)$

\begin{tabular}{|c|c|c|c|}
\hline & $\begin{array}{c}\text { CD } \\
\mathrm{n}(\%)\end{array}$ & $\begin{array}{r}\text { Enf./Méd. } \\
n(\%) .\end{array}$ & Total \\
\hline \multicolumn{4}{|l|}{ Conhecimento sobre câncer bucal } \\
\hline Ótimo e Bom & $13(52)$ & $2(8)$ & $15(60)$ \\
\hline Regular e Ruim & $3(12)$ & $7(28)$ & $10(40)$ \\
\hline \multicolumn{4}{|c|}{ Qual gênero apresenta maior prevalência de câncer de boca? } \\
\hline Masculino & $14(56)$ & $6(24)$ & $20(80)$ \\
\hline Não há diferença entre os gêneros & $2(8)$ & $1(4)$ & $3(12)$ \\
\hline Não sei responder & 0 & $2(8)$ & $2(8)$ \\
\hline \multicolumn{4}{|c|}{ Neoplasia maligna mais prevalente mundialmente na boca $(\mathrm{N}=24)$} \\
\hline Carcinoma de células escamosas & $8(33,4)$ & $2(8,3)$ & $10(41,7)$ \\
\hline Outras & $6(25,0)$ & $5(20,8)$ & $11(45,8)$ \\
\hline Não sabe responder & $2(8,3)$ & $1(4,2)$ & $3(12,5)$ \\
\hline \multicolumn{4}{|c|}{ Faixa etária mais prevalente de câncer de boca } \\
\hline Até 40 anos & $1(4)$ & $2(8)$ & 3 \\
\hline Acima de 40 anos & $13(52)$ & $6(24)$ & 19 \\
\hline Não sei responder & $2(8)$ & $1(4)$ & $3(12)$ \\
\hline \multicolumn{4}{|c|}{$\begin{array}{l}\text { Caracteristicas clínicas que podem ser compativeis com a hipótese de câncer } \\
\text { de boca mais prevalente }\end{array}$} \\
\hline $\begin{array}{l}\text { Lesão ulcerada, coloração eritematosa, } \\
\text { com crescimento lento }\end{array}$ & $6(24)$ & 0 & $6(24)$ \\
\hline $\begin{array}{l}\text { Lesāo exofitica, bordas regulares bem } \\
\text { delimitadas, crescimento rápido }\end{array}$ & 0 & $1(4)$ & $1(4)$ \\
\hline $\begin{array}{l}\text { Lesão endofitica, coloração heterogênea, } \\
\text { cicatrização rápida }\end{array}$ & $1(4)$ & 0 & $1(4)$ \\
\hline $\begin{array}{l}\text { Lesão nodular, coloração homogênea, } \\
\text { crescimento lento }\end{array}$ & $1(4)$ & 0 & $1(4)$ \\
\hline $\begin{array}{l}\text { Lesão infiltrativa, bordas endurecidas, } \\
\text { crescimento rápido }\end{array}$ & $7(28)$ & $4(16)$ & $11(44)$ \\
\hline Não sei responder & $1(4)$ & $4(16)$ & $5(20)$ \\
\hline \multicolumn{4}{|c|}{ Capacidade de identificar as lesōes potencialmente malignas $(\mathrm{N}=24)$} \\
\hline Bom & $9(37,5)$ & $1(4,2)$ & $10(41,7)$ \\
\hline Regular ou Ruim & $7(29,2)$ & $7(29,2)$ & $14(58,3)$ \\
\hline
\end{tabular}

Em relação ao estadiamento clínico do câncer de boca, 28\% participantes não souberam responder quanto a sua finalidade. No entanto, entre os profissionais, $60 \%$ responderam que um dos itens avaliados no estadiamento é o tamanho da lesão, e $52 \%$ que a estimativa de metástase tanto linfonodal quanto à distância também são analisados (Tabela 4).

A maior parte dos profissionais $88 \%$ expressou a vontade de participar de algum curso de atualização sobre o câncer bucal.
Tabela 3 - Atitude/Conduta clínica a respeito do câncer bucal. Cirurgião-dentista, Enfermeiro, Médico. Anápolis-GO, 2021. (N=25)

\begin{tabular}{|c|c|c|c|}
\hline & $\begin{array}{c}\text { CD } \\
\mathrm{n}(\%)\end{array}$ & $\begin{array}{l}\text { Enf./Méd. } \\
\text { n (\%) }\end{array}$ & Total \\
\hline \multicolumn{4}{|c|}{$\begin{array}{l}\text { Você costuma orientar seus pacientes sobre os fatores de risco } \\
\text { predisponentes ao câncer bucal? }\end{array}$} \\
\hline Sim, todos os pacientes & $5(20)$ & $1(4)$ & $6(24)$ \\
\hline $\begin{array}{l}\text { Sim, apenas paciente que apresentam } \\
\text { algum fator de risco }\end{array}$ & $11(44)$ & $4(16)$ & $15(60)$ \\
\hline Não & 0 & $4(16)$ & $4(16)$ \\
\hline \multicolumn{4}{|c|}{ Já diagnosticou pacientes com câncer bucal? } \\
\hline Sim & $12(48)$ & $1(4)$ & $13(52)$ \\
\hline Não & $4(16)$ & $7(28)$ & $11(44)$ \\
\hline Não atuo em prática clínica & 0 & $1(4)$ & $1(4)$ \\
\hline \multicolumn{4}{|c|}{ Estou adequadamente capacitado para realizar uma biopsia } \\
\hline Concordo & $2(8)$ & $1(4)$ & $3(12)$ \\
\hline Discordo & $14(56)$ & $5(20)$ & $19(76)$ \\
\hline Não atuo em prática clínica & 0 & $3(12)$ & $3(12)$ \\
\hline \multicolumn{4}{|c|}{ Biópsia de lesão suspeita de câncer de boca em tecido mole } \\
\hline Incisional & $5(20)$ & 0 & $5(20)$ \\
\hline Excisional| & $8(32)$ & 0 & $8(32)$ \\
\hline Não sei responder & $3(12)$ & 9 & $12(48)$ \\
\hline
\end{tabular}

Tabela 4 - Conhecimento sobre o câncer bucal. Anápolis-GO, 2021. ( $\mathrm{N}=25)$

\begin{tabular}{lc}
\hline Finalidade do estadiamento clínico do câncer de boca & $\mathbf{n}(\%)$ \\
\hline $\begin{array}{l}\text { Uniformidade clínica (facilitando a comunicação entre os } \\
\text { profissionais de saúde). }\end{array}$ & $11(44)$ \\
Planejamento do tratamento. & $18(72)$ \\
Indicação de prognóstico. & $14(56)$ \\
Ajudar na avaliação dos resultados do tratamento & $6(24)$ \\
Estabelecer diagnóstico & $9(36)$ \\
Não sei responder & $7(28)$ \\
\hline Itens avaliados no estadiamento do câncer de boca & \\
\hline Tamanho da lesão & $15(60)$ \\
Estimativa de metástase linfonodal & $13(52)$ \\
Estimativa de metástase à distância & $13(52)$ \\
Coloração da lesão & $3(12)$ \\
Infiltração da lesão em tecido ósseo & $13(52)$ \\
Consistência da lesão à palpação & $5(20)$ \\
Não sei responder & $9(36)$ \\
\hline
\end{tabular}

\section{DISCUSSÃO}

Embora os cirurgiões-dentistas tenham se autoavaliado com conhecimento ótimo ou bom sobre o câncer bucal, não se 
sentem seguros para realizar a biópsia de lesão suspeita de câncer de boca em tecido mole. Estudos realizados anteriormente no Brasil, mostraram que os cirurgiões-dentistas se autoavaliam com baixo conhecimento e inseguros para realizar procedimentos de diagnóstico do câncer de boca, ${ }^{2,8,9}$ porém apresentam bons conhecimentos a respeito das características básicas da patologia.

Os profissionais enfermeiros e médicos demonstraram ter menor conhecimento e ainda ter grandes dúvidas referentes ao tipo de câncer mais comum, a localização e os principais fatores de risco para a patologia. Destacando-se que o enfermeiro é um profissional apto para realizar o diagnóstico precoce do câncer de boca, por meio da anamnese e do exame físico que, após uma revisão minuciosa de todos os segmentos e regiões corporais, pode identificar alterações anatômicas e fisiopatológicas, como as lesões bucais, indicativas de câncer de boca. ${ }^{3}$

Com relação ao tipo de câncer bucal mais comum e as localizações mais frequentes, a maior parte relataram ser 0 carcinoma de células escamosas ocorrendo em língua e soalho bucal, demonstrando concordância com a literatura; dentre as neoplasias orais o Carcinoma Epidermoide, também chamado de Carcinoma de Células Escamosa ou Espinocelular, caracteriza o câncer de cavidade oral mais comum, representando mais de $90 \%$ dos casos diagnosticados. ${ }^{5}$
Maior parte dos entrevistados relataram ter costume de orientar seus pacientes sobre os fatores de risco predisponentes ao câncer bucal apenas para os pacientes que apresentam algum desses fatores de risco. Divergindo de estudos que demonstram que para o profissional que atua na atenção básica onde o primordial é a prevenção e promoção da saúde, é fundamental que saiba orientar o paciente sobre os fatores de risco e a importância do acompanhamento para a prevenção, como também deve diagnosticar precocemente essa patologia. ${ }^{11}$ Ainda, menos da metade dos entrevistados afirmaram realizar exame dos tecidos moles da cavidade bucal do paciente nas consultas iniciais, mostrando que é uma prática comum apenas para profissionais cirurgiões-dentistas.

Parte dos pesquisados responderam que não há diferença entre os sexos para a prevalência de carcinoma espinocelular, além de parte não saber responder a respeito da faixa etária predominante. Não condizendo com a literatura; percebe-se que o perfil mais acometido pelo câncer bucal são homens acima de 45 anos, que na maioria dos casos apresentam fatores de riscos relevantes, como tabagismo, etilismo e exposição excessiva ao sol. ${ }^{4}$

Quantos aos sítios de maior acometimento para o câncer de boca em lábio, destaca-se que parte considerável dos entrevistados informaram não saber responder, porém a maioria respondeu ser mais comum em 
lábio inferior, concordando com a literatura. Carcinoma do vermelhão do lábio geralmente é encontrado em pessoas de pele clara com exposição crônica à radiação ultravioleta do sol. Essa lesão está geralmente associada à desenvolvimento prévio de queilite actínica. Quase $90 \%$ das lesões estão localizadas no lábio inferior. ${ }^{5}$

Conhecer as características clínicas do câncer bucal é fundamental para realização do diagnóstico diferencial da doença, dessa forma o profissional que atua na unidade básica de saúde deve conhecer todas as características clínicas compatíveis com a hipótese do câncer de boca. ${ }^{1}$ Porém $20 \%$ dos entrevistados nesse estudo não souberam responder à questão: "Assinale a alternativa que apresenta características clínicas que podem ser compatíveis com a hipótese de câncer de boca mais prevalente".

$$
\text { O processo de aprendizagem }
$$
continuada é primordial para que os profissionais estejam sempre atualizados em conhecimentos e habilidades, como por exemplo para o diagnóstico do câncer bucal, na prática clínica deve ser realizado o exame intrabucal para identificação de lesões potencialmente malignas. ${ }^{9}$ No entanto, nenhum dos participantes se considera ótimo quanto a sua capacidade de diagnostico, e apenas $41,7 \%$ se consideraram bom. Já na prática clínica $44 \%$ dos participantes não diagnosticaram nenhum caso de câncer bucal.
Ao abordar se o cirurgião-dentista poderia realizar o tratamento oncológico em paciente diagnosticado com câncer bucal, a maioria relatou não saber responder, apenas $32 \%$ dos entrevistados responderam afirmativo para a questão. No entanto, ao serem questionados sobre $\circ$ que significa estadiamento clínico do câncer bucal, somente uma minoria, cerca de $30 \%$, não souberam responder.

Uma limitação do estudo foi a baixa taxa de resposta, sugere-se que para futuras pesquisas sobre o tema sejam realizadas no município a fim de possibilitar análises estatísticas inferenciais. Os pesquisadores lamentam a baixa adesão à participação do público-alvo na pesquisa proposta. Buscamos justificativas no momento pandêmico onde o aspecto emocional dos profissionais da saúde possa ter contribuído. $^{15}$ A pandemia de Covid-19 também levou à necessidade da coleta de dados por meio de formulário eletrônico, que possivelmente possa ter levado alguns participantes a não adesão por falta de familiaridade com a ferramenta ${ }^{16}$ ou não tenham se sentido suficientemente seguros em participar, embora tenha sido garantido a confidencialidade e a privacidade. Outro fato a ser considerado foi o momento da coleta de dados ter ocorrido durante a troca de gestão municipal, fato que pode ter influenciado na baixa adesão devido a possibilidade de 
existência de vínculos trabalhistas precários. ${ }^{15}$

\section{CONCLUSÃO}

Verificou-se neste estudo que os cirurgiões-dentistas na população estudada apresentaram melhor conhecimento sobre a temática do câncer bucal em relação aos outros profissionais da equipe de saúde, no entanto esses divergiram em alguns conhecimentos básicos e ainda se sentem inseguros em realizar procedimentos de biópsia. Ressalta-se neste estudo, a necessidade de educação permanente e continuada a todos os profissionais, a fim de que conhecendo, sejam capazes de realizar diagnóstico precoce e orientar sobre fatores de risco, e possam atuar na redução da morbidade e das consequências graves desta patologia.

\section{AGRADECIMENTOS}

Ao Programa de Bolsa de Iniciação Científica (PBIC) do Centro Universitário de Anápolis por incentivar e apoiar a pesquisa.

\begin{abstract}
Aim: To analyze the knowledge of dentists, doctors and nurses from the Family Health Units of Anápolis-GO, regarding oral cancer. Methods: Quantitative study with doctors, nurses and dentists from family health teams in the city of Anápolis-GO. Data collection took place through the Google Forms application. Data were collected on the demographic profile, knowledge about prevention, diagnosis and treatment, and attitudes and practices regarding the topic. Data were collected between 11/2020 and 02/2021, tabulated and analyzed using descriptive statistics, using the IBM SPSS 22.0 software. Results: Response rate of $11.6 \%(N=25)$, $80 \%$ female, mean age of 36.4 years $(S D=12.0)$, graduation time between zero and 34 years. $60 \%(n=15)$ consider their level of knowledge about oral cancer to be excellent or good. As for the highest prevalence, $80 \%(n=20)$ mentioned the male gender, aged over 40 years $76 \%(n=19)$ and $40 \%(n=10)$ answered that squamous cell carcinoma is the most prevalent neoplasm in the mouth. $20 \%(n=5)$ of the participants answered that they did not know about the most prevalent clinical characteristics compatible with the hypothesis of oral cancer and the majority $76 \%(n=19)$ did not feel able to perform a biopsy. Most of the participants $88 \%(n=22)$ expressed the desire to participate in an update course on the topic. Conclusions: Professionals know about mouth cancer although they feel insecure in face of some specificities and express a desire for training on the subject.
\end{abstract}

KEYWORDS: Mouth Neoplasms. Knowledge. Personal de Salud. Primary Health Care.

\section{REFERÊNCIAS}

1. Brito $\mathrm{PH}$, Vitalino-Filho $\mathrm{F}$, Alves-Silva $\mathrm{EG}$, Veloso HHP, Cerqueira IKM, Moura LM. Importância do diagnóstico precoce do câncer bucal e conduta adequada do cirurgião-dentista na atenção básica: revisão integrativa. Odontol. Clín.-Cient. 2020, 19(4) 327-32.

2. Oliveira, SRS, Gonzaga AKG. Câncer de boca: avaliação do conhecimento de cirurgiõesdentistas da Estratégia de Saúde da Família de
Mossoró, Rio Grande do Norte. Revista Ciência Plural. 2020, 6(3): 137-53.

3. Amorim NGC, Souza AS, Alves SM. Prevenção e diagnóstico precoce do câncer bucal: Uma revisão de literatura. Revista Uningá. 2019; 56(2): 70-84.

4. Domingos PAS, Passalacqua MLC, Oliveira ALBM. Câncer bucal: um problema de saúde pública. Revista de Odontologia da 
Universidade Cidade de São Paulo. 2017, 26(1): 46-52.

5. Neville BW, Damm DD, Allen CM, Chi AC. Patologia oral \& maxilofacial. 4. Ed. Rio de Janeiro: Elsevier, 2016. 338p.

6. Brasil. Ministério da Saúde. Instituto Nacional do Câncer. Câncer de Boca [homepage]. Rio de Janeiro, RJ; 2020 [acesso em 06 abr. 2021]. Disponível em: https://www.inca.gov.br/tiposde-cancer/cancer-de-boca.

7. Nascimento EPA, Nogueira LT, Silva TSO, Ferreira RS, Pinheiro COB. Câncer bucal: conhecimento de cirurgiões-dentistas e acadêmicos de Odontologia. R. Interd. 2014, 7(3): 9-16.

8. Barros ATOS, Silva CCDC, Santos VDCBD, Panjwani CMBRG, Barbosa KGN, Ferreira SMS. Conhecimento dos cirurgiões-dentistas sobre câncer de boca e orofaringe: uma revisão integrativa. Revista Brasileira de Enfermagem. 2021, 74(1): 1-8.

9. Barros ATOS. Impacto de uma intervenção educativa no conhecimento de cirurgiõesdentistas do sistema único de saúde de alagoas sobre câncer de boca e orofaringe. Maceió. Dissertação [Mestrado em Saúde] - Centro Universitário CESMAC; 2020.

10. Jnaneswar A, Goutham BS, Pathi J, Kunal Jha K, Kumar VSG. A cross-sectional survey assessing knowledge, attitude, and practice regarding oral cancer among private medical and dental practitioners in Bhubaneswar City. Indian J Med Paediatr Oncol. 2017, 38(2):1339.

11. Tomé-Júnior, V, Pinheiro APS, Rathjechamon K, Oliveira MLM, Silva AP, Vieira HGP. Câncer bucal: a importância do diagnóstico precoce. Revista Eletrônica de Ciências Jurídicas, 2019.

12. Santos LCO, Batista OM, Cangussu MCT. Caracterização do diagnóstico tardio do câncer de boca no estado de Alagoas. Braz J Otorhinolaryngol. 2010, 76(4): 416-22.

13. Nery MW. Conhecimento de estudantes de Medicina, Odontologia e Enfermagem sobre o câncer de boca: estudo na cidade de Recife/PE. Recife. Dissertação [Mestrado em Odontologia] - Universidade Federal de Pernambuco; 2018.

14. Vasconcelos EM. Comportamento dos cirurgiões-dentistas das unidades básicas de saúde do município de São Paulo quanto à prevenção e ao diagnóstico precoce do câncer bucal. 2006. Tese de Doutorado. Universidade de São Paulo.

15. Teixeira CFS, Soares CM, Souza EA, Lisboa ES, Pinto ICM, Andrade LR, et al. A saúde dos profissionais de saúde no enfrentamento da pandemia de Covid- 19. Ciência \& Saúde Coletiva. 2020, 25(9): 3465-74.

16. Vasconcellos-Guedes L.; Guedes LFA. Esurveys: Vantagens e Limitações dos Questionários Eletrônicos via Internet no Contexto da Pesquisa Científica. In: X SemeAd - Seminário em Administração FEA/USP (São Paulo, Brasil) [Internet]. 2007 [acesso em 06 abr. 2021]. 